\title{
DISPONIBILIDADE E VALOR NUTRITIVO DA VEGETAÇÃO DE CAATINGA NO SEMIÁRIDO NORTE RIOGRANDENSE DO BRASIL
}

\begin{abstract}
C. SOUZA', H. F. BARRETO', V. GURGEL ${ }^{1}$ e F. COSTA ${ }^{1}$
${ }^{1}$ Instituto Federal de Educação, Ciência e Tecnologia do Rio Grande do Norte - Campus Apodi cicilia.silva@ifrn.edu.br - felipe.barreto@ifrn.edu.br - valnir.gurgel@gmail.com - francisco_per08@hotmail.com

Artigo submetido em fevereiro/2013 e aceito em junho/2013

\section{RESUMO}

O objetivo desse trabalho foi avaliar a disponibilidade, valor nutritivo e a qualidade dos estratos herbáceo, arbustivo e arbóreo de um fragmento da caatinga no sertão do Rio Grande do Norte. Foram traçados 24 transectos imaginários, marcando-se pontos de observação a cada 4 metros, totalizando 576 pontos amostrais. Para amostragem do estrato herbáceo, foi utilizado um quadrado de $1 \mathrm{~m}^{2}$, sendo feita uma projeção de dois metros de altura para a amostragem do estrato arbustivo-arbóreo. Para identificação das espécies, utilizou-se o nome comum e, posteriormente,

obteve-se o nome científico. Foram identificadas 11 espécies, pertencendo a 7 famílias, com predomínio das Leguminosae, sendo 3 arbustivas e 4 arbóreas. A disponibilidade de fitomassa do componente vegetativo variou de 1219,95 (Julho/2010) a 722,28 (Janeiro/2011) kg de matéria seca (MS) por hectare. Apesar da disponibilidade de fitomassa ser relativamente alta no período de transição chuva/seca, apenas uma pequena porcentagem $(10 \%)$ do material encontrado pode ser considerada como forragem, havendo contribuição da serapilheira.
\end{abstract}

PALAVRAS-CHAVE: alimentação, forragem, pastagem nativa, ruminantes.

\section{FODDER AVAIABILITY AND NUTRITIVE VALUE IN THE CAATINGA VEGETATION IN SEMIARID OF BRAZIL}

\begin{abstract}
The objective of this work was to evaluate the availability and quality of the fodder herbaceous, arbustive and arboreous extracts in an fragment of Caatinga, in Rio Grande do Norte State, Brazil. Where drawn 24 were transects, for visual assessment is scored points every four meters, totaling 576 points. For ampling of the herb, we used a square of $1 \mathrm{~m}^{2}$, being made a projection of two feet tall to sample the arbustive-arboreous. For identification of species, we
\end{abstract}

used the common name and then obtained the scientific name. We identified 11 species, belonging to 7 families, with a predominance of Leguminosae, and 3 shrubs and 4 arboreou. The fitomass availability from the herbaceous component varied from 1219.95 (July/2010) to 1089.58 (January/2011) kg of dry matter (DM). Despite the relatively high fitomass availability, only a small percentage (10\%) was considered as fodder from the material found.

KEYWORDS: feeding, forage, native pasture, ruminant. 


\section{DISPONIBILIDADE E VALOR NUTRITIVO DA VEGETAÇÃO DE CAATINGA NO SEMIÁRIDO NORTE RIOGRANDENSE}

\section{INTRODUÇÃO}

A Caatinga é a vegetação predominante na região semiárida do Nordeste, sendo representada por espécies arbustivas, arbóreas e herbáceas, constituindo-se na principal fonte

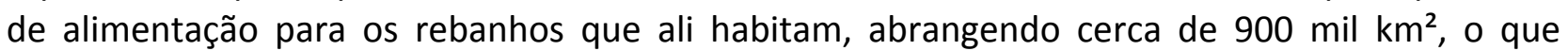
corresponde a aproximadamente $54 \%$ da região Nordeste e $11 \%$ do território brasileiro (Andrade et al., 2006). No entanto, devido à irregularidade na distribuição das chuvas e pulsos de precipitação, a produção animal torna-se bastante vulnerável à estacionalidade da oferta qualitativa e quantitativa de recursos forrageiros, onde no período das águas, a Caatinga rebrota e faz ressurgir o estrato herbáceo, que apresenta uma diversidade de plantas nativas e exóticas naturalizadas, onde cerca de $10 \%$ das plantas podem apresentar características forrageiras, as quais podem ser aproveitadas pelos animais através do pastejo direto.

O conhecimento de alguns parâmetros quantitativos e qualitativos da vegetação, assim como a definição de padrões com os quais a condição das pastagens pode ser avaliada é fundamental para estabelecer um programa de utilização e manejo.

Estudos relatam que na região do Nordeste brasileiro $70 \%$ das espécies botânicas da Caatinga participam significativamente da composição da dieta dos ruminantes durante o período chuvoso, reduzindo sua ocorrência no período seco. No período de estiagem predomina o material remanescente dos vegetais durante o processo de transição do período chuvoso/seco, maximizando a disponibilidade de matéria seca (MS), e minimizando os níveis de digestibilidade.

A determinação da real quantidade de forragem disponível pode ser estimada pela velocidade de crescimento da planta e/ou pelo desempenho animal por meio do controle da quantidade de matéria seca (MS) e nutrientes disponíveis, além de indicações de sua utilização pelo animal e a extensão de seu desperdício. Neste caso, o peso da forragem ou o volume da MS torna-se importante medida de crescimento, permitindo calcular a taxa de lotação e estimar a quantidade de forragem consumida (Estrada et al. 1991).

Apesar da Caatinga apresentar boa disponibilidade de fitomassa no período chuvoso, parte significativa desse material não é utilizada na alimentação dos animais, pois pouco se conhece sobre seu valor nutritivo, o que vem contribuído para a utilização restrita de muitas espécies de valor forrageiro, por isso, tanto as forrageiras nativas como as adaptadas à região têm sido alvo de pesquisas em busca desse conhecimento.

Nesse contexto, considerando-se que a Caatinga é composta de várias famílias botânicas com capacidade de persistir as condições semiáridas do Nordeste, suportando baixas precipitações, além de fornecer biomassa como fonte de energia aos animais, esse trabalho tem como objetivo avaliar as ocorrências vegetais, a disponibilidade e a qualidade da forragem de um fragmento da Caatinga no semiárido brasileiro. 


\section{METODOLOGIA}

A pesquisa foi conduzida em um fragmento de Caatinga de quatro hectares, pertencente ao Instituto Federal de Educação, Ciência e Tecnologia do Rio Grande do Norte - Câmpus Apodi/RN. O período de coleta de dados ocorreu entre os meses de Julho de 2010 a Maio de 2011, sendo as coletas realizadas mensalmente, onde os dados meteorológicos de pluviosidade média e as temperaturas máximas e mínimas foram $6,5 \mathrm{~mm} ; 35,4 \stackrel{\circ}{\circ}$ e $23,3 \stackrel{\circ}{\circ} \mathrm{C}$, respectivamente. O regime térmico é caracterizado por altas temperaturas, com valores máximos concentrados no período de Setembro a janeiro.

Para a caracterização da vegetação na área experimental foi utilizado o método do ranqueamento, onde a área foi dividida em 24 transectos imaginários, no sentido do comprimento e largura, formando uma cruz, totalizando 576 pontos amostrais, de onde foram avaliadas a disponibilidade do extrato arbóreo, arbustivo e herbáceo.

Os dados foram tabulados para dar a proporção dos quadrantes, buscando ranquear as espécies predominantes em $1^{\circ}, 2^{\circ}$ e $3^{\circ}$ lugares, em que as proporções foram multiplicadas por 70,$2 ; 21,1$ e 8,7 respectivamente, e acrescidas para dar as porcentagens do peso bruto de cada espécie (De Vries, 1958).

As amostragens para determinação da ocorrência das espécies foram obtidas a partir da observação de um quadrante de $1 \mathrm{~m}^{2}$, colocados a cada $4 \mathrm{~m}$, sobre um transecto de $50 \mathrm{~m}$, distribuído aleatoriamente dentro do piquete. Esse procedimento foi realizado nos pontos escolhidos a cada período de coleta. Desta maneira, foi obtida uma estimativa da disponibilidade em porcentagem das principais forrageiras existentes na área experimental.

Para a identificação das espécies foi utilizado primeiramente, o seu nome vulgar, sendo posteriormente consultada na literatura para a obtenção do nome cientifico e a que família estas pertence. Foi considerada como fitomassa disponível ao acesso animal aquela vegetação inferior a $2 \mathrm{~m}$ de altura dentro da área de quadrante de $1 \mathrm{~m}^{2}$.

Para a determinação da composição química da vegetação de caatinga, foi recolhido o material vegetativo rente ao solo a cada $12 \mathrm{~m}$ de cada trasnsecto imaginário, incluindo o que se encontrava a dois metros de altura dentro do quadrante. Desta maneira, foi obtida uma estimativa da disponibilidade em peso de forragem e a porcentagem das principais forrageiras existentes na área experimental. Após a coleta, o material foi pesado, e em seguida, realizado uma amostragem composta (média de 100 gramas) para cada período de coleta, pré-seco em estufa de circulação forçada a $55^{\circ} \mathrm{C}$ para futura análise dos nutrientes.

As amostras coletadas foram remetidas ao Laboratório de Nutrição Animal do Centro de Ciências Agrárias da Universidade Federal da Paraíba - CCA/UFPB para as análises de matéria seca $(M S)$, matéria mineral $(M M)$, proteína bruta $(P B)$ e extrato etéreo $(E E)$ em aparelho tipo Soxhlet, segundo o método descrito pela AOAC, 1990; Fibra em detergente neutro (FDN) e fibra em detergente ácido (FDA) (Van Soest, 1985). 
A digestibilidade in vitro da materia seca (DIVMS) foi realizada conforme a metodologia proposta por Tilley e Terry (1963) adaptada por Silva \& Queiroz (2002). O teor de carboidratos totais foi obtido através da fórmula: \%CHOT $=100-(\% \mathrm{~PB}+\% \mathrm{EE}+\%$ Cinzas $)$ e os carboidratos não fibrosos (CNF) pela fórmula: CNF = $100-\left(F D N+P B+2,25^{*} E E\right)$, proposta por Sniffen et al. (1992).

Os dados obtidos a partir do questionário referente à disponibilidade de forragem foram submetidos à análise descritiva por espécie.

\section{RESULTADOS E DISCUSSÃO}

As espécies vegetais que compõem a Caatinga apresentam particularidades específicas em função dos diferentes extratos vegetativos (herbáceo, arbustivo e arbóreo), clima, solo e topografia nas regiões do Nordeste brasileiro. A presença e ocorrência dessas espécies podem ser explicadas pelo fato de possuírem características adaptativas ao tipo de solo existente nessa região do oeste Potiguar, encontrando-se 11 espécies de vegetais presentes na área de estudo, sendo três herbáceas, quatro arbustivas e quatro arbóreas (Tabela 1).

Tabela 1. Nome vulgar, família, nome científico e estrato das espécies encontradas em um fragmento de Caatinga no semiárido Norte Riograndense

\begin{tabular}{l|c|c|c}
\hline \multicolumn{1}{c|}{ NOME VULGAR } & FAMÍLIA & NOME CIENTíFICO & ESTRATO \\
\hline CAATINGUEIRA & Leguminosae & Caesalpinia Pyramidalis Tul. & Arbóreo \\
\hline CAPIM PANASCO & Gramineae & Aristida adscensionis L. & Herbáceo \\
\hline JITIRANA & Concolvulaceae & Ipomoeia $s p$ & Herbáceo \\
\hline JUCÁ & Leguminosae & Caesalpinia ferrea & Arbóreo \\
\hline JUREMA & Leguminosae & Mimosa $s p$ & Arbustivo \\
\hline MALVA-BRANCA & Malvaceae & Herissantia crispa (L.) Briz & Herbáceo \\
\hline MARMELEIRO & Euphorbiaceae & Croton sonderiamus Mull. Arg. & Arbustivo \\
\hline MOFUMBO & Combretaceae & Combretum leprosum Mart. & Arbustivo \\
\hline MORORÓ & Leguminosae & Bauhinia Cheilantha (Bonq.) D. Dietr & Arbustivo \\
\hline PEREIRO & Apocynaceae & Aspidosperma pyrifolium Mart. & Arbóreo \\
\hline SABIÁ & Leguminosae & Mimosa caesalpinifolia & Arbóreo \\
\hline
\end{tabular}

Tabarelli et al. (2000), citam que apesar de a Caatinga ser um dos ambientes menos estudados do Brasil, com aproximadamente $40 \%$ da área ainda não amostrada e $80 \%$ subamostrada, são conhecidas, atualmente, 932 espécies de plantas, das quais 380 são endêmicas desse ambiente. A minoria das espécies que na atualidade foram catalogadas, inclusive as identificadas neste estudo são comuns em diversas áreas de caatinga em diferentes estados e municípios do Nordeste brasileiro, como por exemplo, Catingueira, Pereiro, Sabiá, Malva e outros, no entanto, na maioria das vezes a ocorrências dessas plantas se diferem de região para região. 
A freqüência dos estratos vegetativos expressas em porcentagem (Figura 1) representa a maior frequência média do Marmeleiro (15,47\%), seguida da Malva-branca (13,37\%), Caatingueira (7,94\%) e Jitirana (6,47\%). Esses resultados foram semelhantes aos encontrados por Gonzaga, 2007.

Esse comportamento pode ser explicado pelo fato da vegetação de caatinga nesta área encontrar-se em processo de degeneração, apresentando um solo sem vegetação (desnudo), ocasionado pela ação extrativista e chuvas de alta intensidade concentradas em curto período do ano, além da predominância das espécies forrageiras mais resistentes, como é o caso do Panasco, e outras que não foram identificadas. Além disso, há relatos de que o maior ou menor número de espécies nos levantamentos ocorre em resposta a fatores relacionados à topografia, classe, profundidade e permeabilidade do solo.

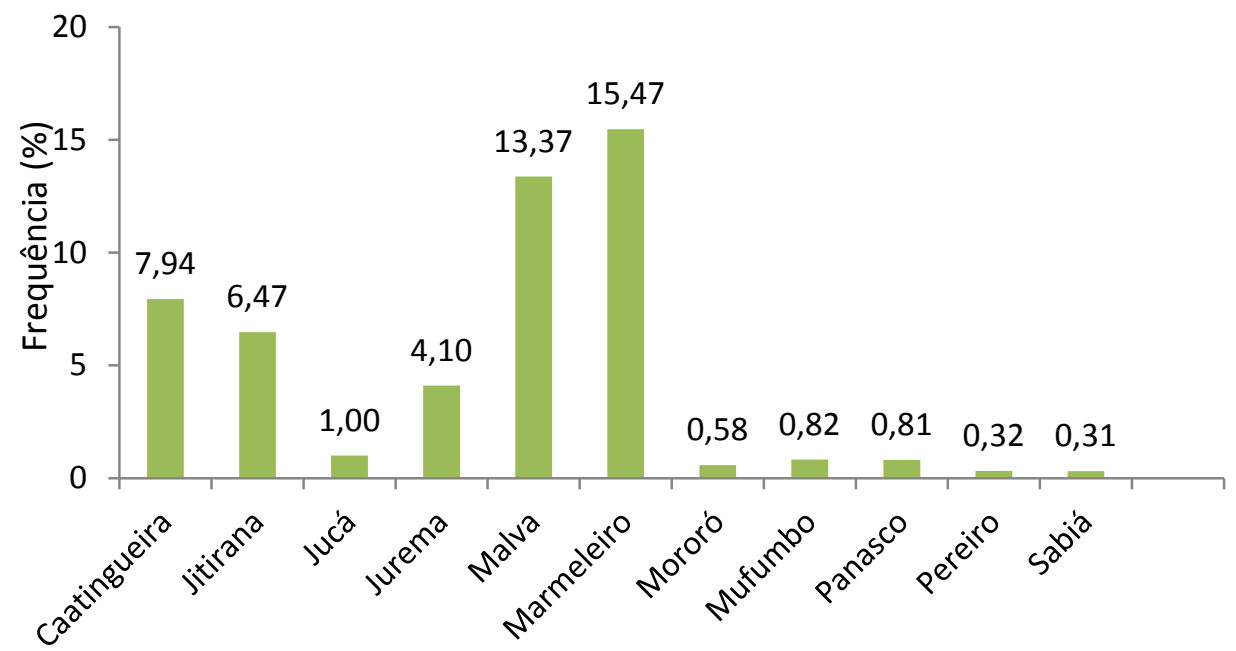

Figura 1: Frequência dos tipos de estratos em porcentagem (\%) dos vegetais encontradas em um fragmento de Caatinga

É importante frisar que os componentes predominantes da vegetação são do tipo arbustivo (20,98\%) e herbáceo (20,66\%) (Figura 2) com dominância de poucas espécies arbóreas. Na mesma figura, observa-se que há uma freqüência significativa de um estrato que neste estudo foi considerado como outros, pelo qual corresponde ao litus ou serrapilheira, material remanescente dos vegetais durante o processo de transição do período chuvoso para o seco, contribuindo para a maximização da disponibilidade de matéria secas e nutrientes, que por sua vez, servirão de alimento para o rebanho que esteja em pastejo, além de servir de fonte de matéria orgânica ao solo.

Os resultados encontrados neste estudo corroboram com Moreira et al. (2006), onde citam que durante a estação das chuvas, a maior parte da forragem é proporcionada pelo estrato herbáceo, com baixa participação da folhagem de árvores e arbustos. No entanto, à medida que a estação seca avança, a folhagem das espécies lenhosas passa a constituir a principal fonte de forragem para os animais. 


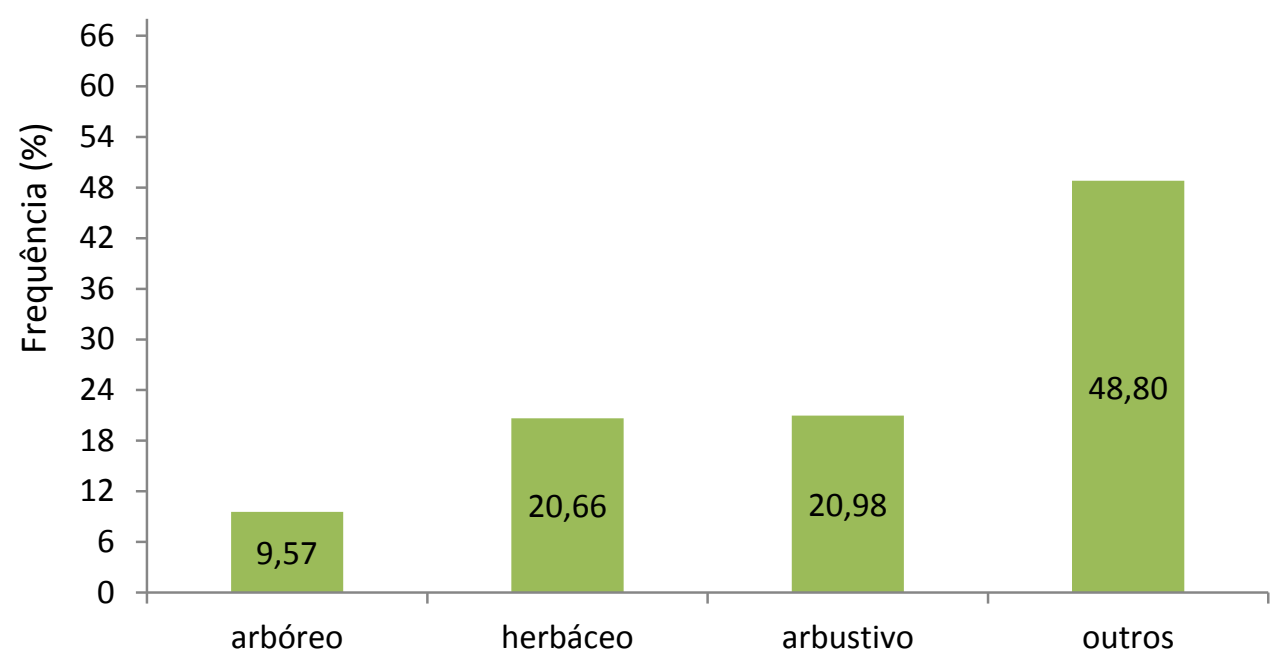

Figura 2: Frequência do estrato herbáceo dos vegetais encontrados em um fragmento de Caatinga

A ocorrência das plantas e a disponibilidade de forragem ( $\mathrm{kg} \mathrm{MS} / \mathrm{ha}$ ) estão representadas na Tabela 2, onde estas variáveis aumentaram do período de Julho para Setembro, reduzindo então em Novembro, mês onde a região encontra-se totalmente seca.

Quanto à disponibilidade de forragem das espécies (kg MS/ha), em termos quantitativos, nota-se a predominância média da Malva-branca $(142,37)$ e Marmeleiro $(177,15)$ e baixa presença ou ausência do Capim Panasco, Jucá, Mororó, Pereiro e Sabiá, que representaram média de 1,$38 ; 3,78 ; 5,62 ; 0,0$ e 0,91 kg MS/ha, respectivamente, em função da baixa ou quase ausência de chuvas no período.

O fato da disponibilidade ( $\mathrm{kg} \mathrm{MS} / \mathrm{ha}$ ) das espécies vegetais encontradas na vegetação de Caatinga ter sofrido comportamento semelhante ao verificado pela sua ocorrência, pode ser explicada pela sua adaptabilidade e resistência.

A classificação denominada como outros, é correspondente ao chamado litus ou serrapilheira, que foi quantificado entre os meses de Julho a Setembro, com disponibilidade variando de 1219,95 a 1088,29 kg MS/ha, respectivamente.

Durante o mês de Janeiro não foi observado à disponibilidade do litus ou serrapilheira (Tabela 2), indicando haver provável retorno de disponibilidade de material verde em função das conhecidas chuvas de verão. Esta situação pode ser explicada pelo comportamento de alguns dos vegetais estudados (Jitirana, Jurema, Marmeleiro e Mofumbo) ao longo dos meses, que reduziram a sua disponibilidade entre Julho e Novembro de 2010, voltando a maximizar ou sofrer pouca variação no mês de Janeiro de 2011. 
Tabela 2. Ocorrência (\%) e disponibilidade (kg MS/ha) de espécies vegetais de um fragmento de Caatinga no semiárido Norte Riograndense

\begin{tabular}{|c|c|c|c|c|c|c|c|c|}
\hline \multirow{3}{*}{$\begin{array}{l}\text { NOME VEGETAL } \\
\text { (VULGAR) }\end{array}$} & \multicolumn{4}{|c|}{ OCORRÊNCIA } & \multicolumn{4}{|c|}{ DISPONIBILIDADE } \\
\hline & \multirow[b]{2}{*}{ JUL } & \multicolumn{2}{|c|}{2010} & \multirow{2}{*}{$\begin{array}{l}2011 \\
\text { JAN }\end{array}$} & \multicolumn{3}{|c|}{2010} & \multirow{2}{*}{$\begin{array}{r}2011 \\
\text { JAN }\end{array}$} \\
\hline & & SET & NOV & & JUL & SET & NOV & \\
\hline CAATINGUEIRA & 5,05 & 2,44 & 2,47 & 1,83 & 61,40 & 24,12 & 26,84 & 23,99 \\
\hline CAPIM PANASCO & 0,0 & 0,25 & 0,0 & 0,0 & 0,0 & 2,50 & 0,0 & 3,03 \\
\hline JITIRANA & 0,89 & 7,30 & 1,82 & 5,00 & 10,14 & 72,29 & 19,83 & 112,71 \\
\hline JUCÁ & 0,0 & 1,44 & 0,0 & 0,19 & 0,0 & 14,26 & 0,0 & 0,88 \\
\hline JUREMA & 2,00 & 6,14 & 4,50 & 3,74 & 23,21 & 60,80 & 49,04 & 51,56 \\
\hline MALVA-BRANCA & 13,32 & 23,47 & 9,03 & 9,52 & 162,18 & 232,29 & 98,13 & 76,89 \\
\hline MARMELEIRO & 12,19 & 0,0 & 12,07 & 40,43 & 148,43 & 0,0 & 131,25 & 428,94 \\
\hline MOFUMBO & 1,16 & 0,38 & 0,0 & 1,59 & 14,10 & 3,74 & 0,0 & 22,97 \\
\hline MORORÓ & 0,83 & 1,11 & 0,0 & 0,0 & 10,15 & 11,03 & 0,0 & 1,31 \\
\hline PEREIRO & 0,0 & 0,0 & 0,0 & 0,32 & 0,0 & 0,0 & 0,0 & 0,0 \\
\hline SABIÁ & 0,32 & 0,0 & 0,0 & 0,0 & 3,67 & 0,0 & 0,0 & 0,0 \\
\hline OUTROS $^{1}$ & - & - & - & - & 786,667 & 570,77 & 763,20 & 0,0 \\
\hline TOTAL & - & - & - & - & 1219,95 & 991,80 & 1088,29 & 722,28 \\
\hline
\end{tabular}

As variáveis qualitativas das principais espécies observadas se mostraram homogêneas para os nutrientes avaliados em função dos diferentes períodos de avaliação (Tabela 3).

Tabela 3. Composição química da disponibilidade total da pastagem em fragmento de Caatinga no semiárido brasileiro

\begin{tabular}{lccccc}
\hline \multirow{2}{*}{\multicolumn{1}{c}{ NUTRIENTES (\%) }} & \multicolumn{5}{c}{ PERÍODOS } \\
\cline { 2 - 6 } & \multicolumn{5}{c}{2010} \\
\cline { 2 - 6 } & JULHO & SETEMBRO & NOVEMBRO & JANEIRO & MÉDIA \\
\hline MATÉRIA SECA & 89,53 & 72,78 & 76,84 & 79,94 & 79,77 \\
MATÉRIA MINERAL & 10,40 & 13,35 & 12,13 & 17,10 & 13,24 \\
MATÉRIA ORGÂNICA & 89,60 & 86,65 & 88,13 & 87,54 & 87,98 \\
PROTEÍNA BRUTA & 7,73 & 7,25 & 7,64 & 8,54 & 7,79 \\
EXTRATO ETÉREO & 3,52 & 2,93 & 5,40 & 5,30 & 4,28 \\
FIBRA EM DETERGENTE NEUTRO & 76,26 & 76,79 & 68,46 & 71,00 & 73.12 \\
FIBRA EM DETERGENTE ÁCIDO & 72,08 & 71,84 & 60,58 & 67,87 & 68,09 \\
CARBOIDRATOS TOTAIS & 78,35 & 76,47 & 74,87 & 69,07 & 74,69 \\
CARBOIDRATOS NÃO FIBROSOS & 8,09 & 9,37 & 11,84 & 8,56 & 9,46 \\
DIGESTIBILIDADE “IN VITRO” & 55,00 & 47,50 & 44,70 & 56,00 & 50,80 \\
\hline
\end{tabular}

Os valores médios de proteína bruta (PB) observados nesse levantamento são considerados bons, já que este resultado trata-se de uma vegetação nativa de caatinga em fase de transição do período chuvoso (Julho/2010) para o período seco (setembro e novembro/2010), alcançando ainda as conhecidas chuvas de verão (janeiro/2011), onde foi superior a $7 \%$ na matéria seca, que para dietas dos ruminantes é considerado por Minson (1990) o valor mínimo para que ocorra crescimento da microflora microbiana, em que animais submetidos à pastejo nessas áreas alcançam níveis de consumo e digestibilidade suficientes para sua manutenção. 
Contudo, o grau da maturação das plantas encontradas no fragmento da Caatinga, podem promover a redução do teor de proteína bruta e aumentar a lignina, que por sua vez, contribui para a maximização dos teores de fibra em detergente neutro (média de 73,12\%) e fibra em detergente ácido (média de 68,09\%), possibilitando redução na digestibilidade dos nutrientes $(50,80 \%)$.

Esses resultados foram semelhantes aos adquiridos por Moreira et al. 2006, ao caracterizar a vegetação de Caatinga no sertão de Pernambuco durante o período chuvoso, exceto para a digestibilidade, que no presente estudo foi superior.

Apesar dos resultados obtidos, é fundamental conhecer a composição química da dieta selecionada pelos ruminantes nas áreas de pastejo, especialmente de caprinos, já que estes podem selecionar dietas que atendam suas demandas nutricionais de acordo com a qualidade da fitomassa disponível. Dessa maneira, a tomada de decisão para um planejamento de tornar os sistemas de produção mais eficaz é válida, bem como o efeito do pastejo sobre a comunidade vegetal, melhorando programas de suplementação alimentar.

\section{CONCLUSÕES}

A ocorrência de 11 espécies de plantas, que se classificam entre 3 herbáceas, 4 arbustivas e 4 arbóreas, encontradas no fragmento de uma área de caatinga no semiárido do Rio Grande do Norte durante a época das águas e seca, as plantas Marmeleiro, malva-branca, Catingueira e Jitirana apresentam-se predominantes; que quando associadas à disponibilidade da liteira, contribuem como fitomassa, sendo consideradas de potencial forrageiro.

\section{REFERÊNCIAS BIBLIOGRÁFICAS}

1. ANDRADE, A. P.; SOUSA, E.S.; SILVA, D.S.; SILVA, I.F.; LIMA, J.R.S. Produção Animal no Bioma Caatinga: Paradigmas dos 'Pulsos - Reservas'. Revista Brasileira de Zootecnia, João Pessoa/PB, v. 35, n. Suplemento, p. 138-155, 2006.

2. DE VRIES, D. M. 1958. Methoden van botanisch graslandonderzock en graslandwaardering. Lecture Given at Wageningen, Netherlands (Holanda), 25 May, (Mimco), 1958.

3. eSTRADA, C.L.H. NASCIMENTO JR., D., REGAZZI, A.J. Efeito do número e tamanho do quadrado nas estimativas pelo Botanal da composição botânica e disponibilidade de matéria seca de pastagens cultivadas. Revista Brasileira de Zootecnia, Viçosa/MG, v. 20, n.5, p.483-493, 1991.

4. GONZAGA, A.R. Estimativa de Consumo e Degradabilidade da Extrusa em Caprinos Suplementados na Caatinga. 2007. 106f. Dissertação (Mestrado em Produção Animal) Universidade Federal da Paraíba/Centro de Ciências Agrárias. 2007. 
5. MINSON, D.J.; STOBBS, T.H.; HEGARTY, M.P. et al. Measuring the nutritive value of pasture plants. In: SHAW, N.H.; BRYAN, W.W. (Eds.) Tropical Pasture Research Principles and methods. Bulletin, 51. p.308-337, 1976.

6. MOREIRA, N.J.; LIRA, M.A.; SANTOS, M.V.F.; FERREIRA, M.A.; ARAUJO, G.G.L.; FERREIRA, R.L.C. Caracterização da vegetação de Caatinga e da dieta de novilhos no Sertão de Pernambuco. Pesquisa Agropecuária Brasileira, Brasília/DF, v.41, n.11, p.1643-1651, 2006.

7. REIS, R. A., DA SILVA, S.C. Consumo de forragens. IN: Berchielli, T.T., Pires, A.V., Oliveira, S. g. Nutrição de Ruminantes. FUNEP, Jaboticabal, 2006.

8. SILVA, D.J.; QUEIROZ, A.C. Análises de alimentos - métodos químicos e biológicos. 3.ed. Viçosa: UFV, 2002. 235p.

9. TABARELLI, M.; SILVA, J.M.C.; SANTOS, A.M.M. Análise de representatividade das unidades de conservação de uso direto e indireto na Caatinga: análise preliminar. 2000. In: SILVA, J.M.C.; TABARELLI, M. (Coord.) WORKSHOP Avaliação e identificação de ações prioritárias para conservação, utilização sustentável e repartição de benefícios da biodiversidade do bioma caatinga. 13p. Petrolina, 2000. 